le portiQue $\begin{array}{ll}\text { Le Portique } \\ \text { Revue de philosophie et de sciences humaines }\end{array}$

33 | 2014

Straub !

\title{
Antigone s'arrête à Ségeste
}

\section{Olivier Goetz}

\section{OpenEdition}

Journals

Édition électronique

URL : http://journals.openedition.org/leportique/2748

DOI : $10.4000 /$ leportique.2748

ISSN : $1777-5280$

\section{Éditeur}

Association "Les Amis du Portique"

Édition imprimée

Date de publication : 1 mai 2014

ISSN : 1283-8594

\section{Référence électronique}

Olivier Goetz, «Antigone s'arrête à Ségeste », Le Portique [En ligne], 33 | 2014, document 1, mis en ligne le 05 février 2016, consulté le 12 avril 2021. URL : http://journals.openedition.org/leportique/2748 ;

DOI : https://doi.org/10.4000/leportique.2748

Ce document a été généré automatiquement le 12 avril 2021.

Tous droits réservés 


\title{
Antigone s'arrête à Ségeste
}

\author{
Olivier Goetz
}

1 S'il faut admettre, avec Marguerite Duras, que le cinéma arrête l'imaginaire ${ }^{1}$, l'Antigone que Danièle Huillet et Jean-Marie Straub sont allés filmer en Sicile, en 1992, dans les ruines du théâtre de Ségeste, apparait sur l'écran comme arrêtée, au terme d'un long voyage. Le périple de cette très vieille jeune fille commence, en effet, dans « la nuit des temps ", c'est-à-dire, en réalité, dans l'obscurité relative d'une période archaïque où le monde grec élabore oralement ses mythologies. Mais, Antigone elle-même ne devient personnage, à proprement parler, qu'à partir du $\mathrm{V}^{\mathrm{e}} \mathrm{s}$. avant J.-C., lorsque l'évolution des techniques et des mentalités autorise la transformation scénique des «idoles» en « images », pour reprendre la terminologie et le raisonnement de Jean-Pierre Vernant: «Pour que l'idole devienne image, il ne suffit pas que, dégagée du rituel, elle n'assume plus d'autre fonction que d'être vue et, sous le regard de la cité, se transforme en pur spectacle, il faut aussi qu'au lieu d'insérer dans le monde visible la présence de l'invisible divin, elle se propose, par l'imitation experte des formes extérieures du corps, d'en reproduire l'apparence aux yeux des spectateurs $»^{2}$. Telle est bien l'invention du théâtre, mais aussi, potentiellement, celle d'un futur cinéma...

2 Quand bien même un spectateur ferait, aujourd'hui, connaissance de la fille d'ÆEdipe à travers le film des Straub, il sentirait d'emblée qu'Astrid Ofner, la statue vivante qui prête corps et voix au personnage, jouant des transparences et des opacités de la langue allemande ${ }^{3}$, constitue, en réalité, un palimpseste. Au regard du vaste itinéraire qui, depuis la Renaissance, force l'admiration des lettrés ${ }^{4}$, l'Antigone des Straub constitue un raccourci lapidaire, puisqu'elle n'en considère que trois étapes (ou trois strates) :

3 - 442 avant J.-C.: Inscription de la tragédie de Sophocle au concours des grandes dionysies d'Athènes

4 - 1797-1804: Traduction/rédaction, par Hölderlin, de son Antigone d'après Sophocle, entre Bordeaux et Tübingen

5 - 1948: Présentation par Bertolt Brecht de sa propre version d'Antigone, d'après Sophocle/Hölderlin, au Berliner Ensemble.

6 Tout en manifestant un certain attachement à la Grèce (décors et costume, références religieuses et politiques), cette Antigone ne revendique aucune « authenticité » native. 
En effet, le film n'ambitionne ni la reconstitution d'un récit antique dépourvu - par définition -, de tout substrat historique (à la manière d'un péplum hollywoodien), ni la transposition poétique d'un mythe (à la manière de Pasolini); il est, à proprement parler, la mise en scène cinématographique d'une pièce de Brecht, lecteur de Hölderlin, lecteur de Sophocle. C'est-à-dire que, pour prendre les choses dans l'autre sens, Sophocle ne constitue plus ici qu'une convocation, le fantôme lumineux et exalté qui surgit lorsqu'un dramaturge "non-aristotélicien» s'empare, juste après la Seconde Guerre, du texte que le promoteur d'une Grèce idéale et d'une Hespérie utopique écrivit à l'aube du $\mathrm{XIX}^{\mathrm{e}}$ siècle ${ }^{5}$. L'Antigone du film est, très précisément, l'objet de cette appropriation où transparaît, en écho tuilé, l'histoire d'une pensée théâtrale. Pour autant, si Antigone n'est pas filmée à Thèbes, ou dans un décor qui prétende restaurer mimétiquement la cité de Créon, le fait qu'on la (re)trouve sur le site d'un théâtre de l'époque hellénistique (III ${ }^{\mathrm{e}}$ siècle avant J.-C.) remanié, comme tant d'autres, à l'époque romaine ( $\mathrm{I}^{\mathrm{er}}$ siècle avant J.-C.) pourrait recouvrir quelque intention subliminale. Ségeste est bien cette cité qui entraîna, à son corps défendant, la fin de la suprématie d'Athènes ${ }^{6}$. Et, quand bien même la détermination du cadre ne devrait rien qu'à des opportunités circonstancielles ou à la beauté d'un paysage, rien n'empêche le spectateur, tel un touriste zélé parcourant l'Italie un guide à la main, d'enrichir la contemplation des images d'une méditation sur les fata de l'Histoire...

7 Ce qu'on appelle le « théâtre grec » constitue jusqu'à une date récente, pour la culture occidentale, une référence massive et quelque peu terrifiante. De l'édification du théâtre olympique de Vicence (inauguré, en 1585, par une représentation d' $E$ dipe roi ${ }^{7}$ ) aux projets de théâtre populaire qui fleurissent à la fin du XIX siècle et au début du Xxe la Grèce antique fait elle-même office de mythologie originaire. Dans leur désir de réformer un théâtre jugé par eux élitiste et corrompu, Jules Michelet, Romain Rolland, Maurice Pottecher, Edward Gordon Craig, Firmin Gémier, Jacques Copeau et Max Reinhard revendiquent, chacun à sa manière, un retour aux sources grecques qui passe, notamment, par la recherche de formes scéniques innovantes, inspirées par le modèle des théâtres antiques. De ce dispositif primitif, largement fantasmé, les grands réformateurs du théâtre retiennent surtout la forme circulaire de l'orchestra ${ }^{8}$, synonyme, à leur yeux, d'un esprit vraiment démocratique ${ }^{9}$. Il serait facile de montrer, par exemple, que la formule révolutionnaire $d u$ «tréteau nu » défendue par Copeau ${ }^{10}$ découle, malgré les apparences, de ce tropisme originaire et que l'apparente nudité du plateau s'encombre en fait de tout un appareil idéologique... Mais, le lien entre théâtre et politique, ces deux "inventions" athéniennes, est si profondément inscrit dans l'histoire des mentalités qu'il semble constitutif de toute innovation en matière d'esthétique théâtrale. Pour le théâtre moderne (l'expression est redondante, puisque le théâtre, tel qu'on l'entend désormais, est, par définition, une invention moderne), la référence à la "Grèce » constitue un lieu commun dont le caractère dominant et le prestige résistent, jusqu'à ce jour, à toute tentative de déconstruction. Face à un prétendu "miracle grec» (le siècle de Périclès considéré comme le lieu de tous les commencements), il convient, cependant, de faire preuve d'un peu de circonspection. Il s'agit, d'abord, avec Jean Duvignaud, de critiquer l'impérialisme culturel qui entend placer notre conception du théâtre (et, notamment, le genre " tragédie ») au faîte de la hiérarchie du beau. Car, pour ce sociologue du spectacle, le goût de l'Antiquité ne fait, depuis le Moyen Âge, que refléter la nostalgie, voire la frustration des clercs ${ }^{11}$. Il s'agit, également, de contester une lecture essentiellement morale ou littéraire des textes dramatiques grecs, tâche à laquelle s'emploient, depuis quelques décennies, un certain 
nombre d'hellénistes, anthropologues ou historiens de la culture, qui préfèrent considérer la dimension événementielle (ou rituelle) du phénomène théâtral plutôt que d'y voir le lieu d'une production d'œuvres monumentales...

8 Ce disant, s'éloigne-t-on tellement de l'entreprise des Straub, si cultivée et si respectueuse de la littéralité des œuvres? Peut-être convient-il, pour en juger, d'adopter naïvement le point de vue de ce public improbable qui aborderait Antigone à travers leur film. Et tenter d'oublier, autant que faire se peut, les interprétations plaquées, au cours des siècles, sur un "chef-d'œuvre" antique dont la signification, autant que l'usage, nous échappe largement. Car, non seulement on ignore, pour l'essentiel, de quelle manière et dans quel contexte il fut primitivement représenté, mais on peut même douter qu'il ait fait l'objet d'une représentation ou, du moins, que la notion de "représentation" s'applique à ce qui constituait, en réalité, l'expérience directe d'un événement spectaculaire, avant qu'une certaine tradition « classique » ne s'en empare. De sorte qu'on n'appréhende jamais cet objet insaisissable qu'à travers un certain nombre de filtres, à commencer, bien sûr, par celui des diverses traductions. Or, nous devons, avec Nicole Loraux ${ }^{12}$, envisager le théâtre grec comme un "théâtre des voix »; y reconnaitre une forme d'» oratorio » où le son prime sur le sens, la phônè sur le logos. De là à formuler, avec Florence Dupont, l'insignifiance de la tragédie grecque ${ }^{13}$, il n'y a plus qu'un pas que seules nos réticences à abandonner toutes ces couches intermédiaires (en l'occurrence, le communisme de Brecht et le jacobinisme d'Hölderlin) accumulées entre un événement primitif et nous, nous empêcheraient de franchir.

9 La leçon que l'on peut retenir des commentateurs qui, au cours des dernières décennies, se sont attachés à prendre le contre-pied d'une très lourde tradition scolaire, c'est que le théâtre antique n'est pas cette activité éminemment politique que l'on a toujours dite, du moins, s'il s'agit de faire reposer la politique sur le consensus qui définit, en Grèce comme ailleurs, le fonctionnement de la démocratie. En témoigne le fait que la chorégie, à Athènes, ne se pratique pas sur l'Agora ${ }^{14}$, mais dans un espace dévolu aux dieux, et, plus précisément, à Dionysos. C'est sur le flanc de l'Acropole, en marge de l'astu (ou de la polis), que se déroule l'activité théâtrale et, là, le peuple (contrairement à celui de l'Assemblée) n'est pas acteur, il n'est que spectateur. La tragédie n'est pas politique parce qu'elle introduit une crise qui menace l'ordre civique. Le fait que les femmes y prennent la parole est le signe le plus patent de cette menace. Exclues de la citoyenneté (et du champ politique en général), les femmes de la société grecque sont avant tout des mères et des nourrices. Le maintien de l'ordre public exige que leur deuil soit maitrisé ${ }^{15}$. Or, complaisamment exprimé, le deuil des héroïnes tragiques a pour caractéristique de demeurer inconsolable... Et, si la tragédie dit quelque chose, c'est que ceux qui, au nom du politique, rappellent à l'ordre les endeuillées sont rien de moins que des assassins : Clytemnestre pour Électre, Créon pour Antigone...

Face aux commentaires qui, d'un côté, privilégient une interprétation morale de la tragédie, ou qui, de l'autre, insistent sur son caractère rituel incompréhensible, il convient, peut-être, de défendre la thèse qu'elle constitue l'expression conflictuelle de la tension qui existe, précisément, entre les significations du discours et la dimension sonore de la plainte... Cela ne permet pas de dire du théâtre grec qu'il est apolitique, mais plutôt, comme le souligne encore Nicole Loraux, qu'il est anti-politique. Antipolitique, la tragédie reste évidemment politique (tout comme l'anti-psychiatrie 
des années 1970 était encore psychiatrique), mais dans le sens de l'anti-; elle propose une autre politique, une politique autre.

Il revient à Sophocle d'avoir dégagé d'une mythologie claire-obscure des figures dont il met en évidence, grâce au dispositif théâtral, l'absence radicale et définitive de tout substrat réel. Après cela, au fond, Antigone, n'est plus qu'un rôle tenu, à côté de ceux d'Ismène et de Créon, par un acteur ${ }^{16}$. Et, au fond, c'est ce dégagement qui permet à Hölderlin, dramaturge innovant et versificateur génial, de continuer à faire, vingt-deux siècles plus tard, dialoguer des voix... Quant à Brecht, n'est-ce pas, précisément, la polyphonie et la polysémie qui lui servent d'outils pour lutter contre l'envoûtement dramatique auquel s'oppose sa propre conception du théâtre? De même que, lorsqu'il conçoit le Lehrstück intitulé «Celui qui dit oui» (texte qui prône le sacrifice d'un enfant au profit d'un bien collectif), la voix antagoniste des jeunes acteurs pour qui il écrit le pousse à concevoir, presque aussitôt, « Celui qui dit non ", non pour invalider la version primitive à laquelle cette seconde version s'oppose, mais pour éviter, précisément, que le texte se fige dans une univocité tragique ${ }^{17}$. La grandeur de Brecht reste précisément d'être parvenu à produire un outil réflexif qui, sans nier l'impact émotionnel (et, pourquoi pas? cathartique) de la représentation, et, nonobstant ses propres convictions, refuse d'inféoder l'écriture théâtrale à une doctrine ou à une idéologie.

Dans Antigone (le film), Danièle Huillet et Jean-Marie Straub font jouer Antigone (le personnage) dans ce que les Grecs appelaient un theatron. Ce mot, on le sait, n'est pas équivalent de notre " théâtre " puisqu'il désigne, non une activité, mais un lieu et qu'il est dépourvu de toute connotation «littéraire». En opérant ce qui ressemble bel et bien à un retour d'Antigone dans les vieilles pierres de la Sicile contemporaine, le film de Danièle Huillet et Jean-Marie Straub fait, certes, entendre la voix des poètes (Brecht et, en échos tuilés, Hölderlin et Sophocle), mais il le fait dans un environnement qui jette sur la lettre du texte un jour particulier. En rendant à la pensée théâtrale d'Antigone, non pas son espace primitif (définitivement oublié ou perdu ${ }^{18}$ ), mais la trace ruinée, transformée, cicatrisée de sa disparition, en offrant pour véhicule à Antigone la terre, l'herbe et le ciel d'un coin de Sicile miraculeusement préservé, le cinéma déplace le public hors des commentaires dont le théâtre occidental est resté si longtemps encombré. Si, l'opposition théâtre/cinéma est l'une des questions qui traverse, de part en part, le cinéma des Straub, Antigone peut servir de modèle à la manière dont ils résolvent ce conflit interne de la représentation. Le parti-pris n'est pas an-historique, puisque l'histoire est largement citée (choix du lieu, choix des costumes, choix de la fable). Mais ces éléments sont contextualisés, placés au plus près de sources matérielles (paysages, ruines) qui en ravivent le propos. Sans contredire les données historiques dont il dispose, ce cinéma propose une autre façon de voir les choses ou de lire les livres... Sous les mots d'Antigone (ceux de Sophocle-Hölderlin-Brecht), les Straub retrouvent un peu de "nature vraie», comme disait Antonin Artaud ${ }^{19}$. Le propre de leur cinéma. 


\section{NOTES}

1. . «Le cinéma arrête le texte, frappe de mort sa descendance : l'imaginaire. C'est là sa vertu même : de fermer. D'arrêter l'imaginaire ", Marguerite DURAS, «Le deuxième projet ", Le Camion, Paris, Les Éditions de Minuit, 1977, p. 75.

2. . Jean-Pierre VERnANT, Figures, idoles, masques, Paris, Julliard, 1990, p. 11.

3. . Et ce, quel que soit le pays où l'on voit le film, selon le principe d'un cinéma en prise de son direct dont les dialogues ne sont jamais doublés. La langue des personnages est donc, bel et bien, à prendre en compte.

4. . Voir Georges STEINER, Les Antigone, Paris, Gallimard, 1986.

5. . Voir Pierre BERTAUX, Hölderlin, Essai de biographie intérieure, Paris, Hachette, 1936.

6. . En 416 avant J.-C., après avoir vainement sollicité l'aide d'Agrigente et de Carthage, Ségeste obtint le concours d'Athènes dans sa guerre contre Sélinonte. L'expédition athénienne fut écrasée devant Syracuse en 413, ce qui sonna le glas de son hégémonie sur le monde grec. Un siècle plus tard, Ségeste est détruite par Agathoclès, tyran de Syracuse, et ses habitants sont massacrés. Par la suite, attaquée par les Carthaginois lors de la $1^{\text {ère }}$ guerre punique (264-241 avant J.-C.) la cité est délivrée par les Romains et rattachée à l'Empire. C'est de cette époque que date la construction du fameux théâtre.

7. L'édification de ce théâtre par Palladio, en 1580, sur les plans de l'architecte latin Vitruve, témoigne de la constitution d'une activité théâtrale professionnelle à la Renaissance.

8. . Max Reinhardt (en Allemagne) et Firmin Gémier (en France), notamment, ont tous deux voulu monter Edipe roi dans des cirques, dispositifs circulaires qui leur semblaient présenter des analogies avec la forme du théâtre grec antique.

9. C'est la forme que prend, par exemple, l'hémicycle du Palais Bourbon (aménagé en 1832 par l'architecte Jules de Joly) qui abrite, jusqu'à nos jours, l'Assemblée nationale française.

10. . « Pour un théâtre neuf, qu'on me donne un tréteau nu » dit Jacques Copeau.

11. . "Jouer l'Antiquité par le théâtre, c'est susciter dans l'existence présente le modèle ancien, rendre momentanément actuel un idéal, faire parler des héros anciens. C'est redevenir contemporains de ces héros admirés, se projeter dans l'image d'une Antiquité qui n'est plus tout à fait morte, surmonter le temps. Entreprise systématique de restauration magique que seuls pouvaient réaliser des "complices", unis par la connaissance d'une langue et de textes, d'une écriture et d'une nostalgie, presque d'une frustration». Jean DUVIGNAUD, Les Ombres collectives, Paris, PUF, 1973, p. 130.

12. . Voir Nicole LORAUX, La Voix endeuillée, essai sur la tragédie grecque, Paris, Gallimard, 1999.

13. . Voir Florence DUPONT, L'Insignifiance tragique, Paris, Gallimard, Le Promeneur, 2001.

14. . Voir Nicole LoRAuX, op. cit., p. 28 et suivantes.

15. . Nicole LORAuX (op. cit.) rappelle que législateur Solon, un des «inventeurs » du système démocratique, aurait interdit aux femmes la pratique du thrène lyrique.

16. . Un acteur, et non une actrice, puisque, comme on sait, les rôles féminins étaient tenus, chez les Grecs, par des hommes.

17. . Voir Bertolt BRECHT, Celui qui dit oui, Celui qui dit non, Paris, L'Arche, 1959. «Cette version de l'opéra suscita de vives discussions dans le public même auquel il était destiné [...]. À la suite de ces discussions, Brecht récrivit la même année les deux versions dont nous donnons ici le texte français, et qui l'une et l'autre diffèrent considérablement de la version primitive, la motivation du "oui" ayant été rendue plus concrète en même temps qu'on montrait la possibilité du "non" " (Note du traducteur, p. 215). 
18. Tout porte à croire que le dispositif grec du ve siècle était, en fait, extrêmement modeste : de simples gradins de bois épousant la déclivité d'une colline entouraient un espace circulaire de terre battue (l'orchestra) fermé par la skénè, une simple baraque, également de bois.

19. . « Il a fait, sous la représentation, sourdre un air, et en elle enfermer un nerf, qui ne sont pas dans la nature, qui sont d'une nature et d'un air plus vrais, que l'air et le nerf de la nature vraie ». (Antonin ARTAUD, Van Gogh ou le Suicidé de la société). L'expression de "nature vraie » est reprise, de manière tout aussi pertinente, dans Pour en finir avec le jugement de Dieu.

\section{AUTEUR}

\section{OLIVIER GOETZ}

Olivier Goetz est maître de conférences au département « Arts » de l'Université de Lorraine. Spécialiste en histoire et en esthétique des arts du spectacle, il développe une recherche transversale et pluridisciplinaire dans le domaine de la création artistique, des arts plastiques et des arts du spectacle. 